\title{
Fractality of the penrose tiling in coordination Cayley's tree graphs representation method
}

\author{
V. Yudin* \\ Far-East National University E-mail: mih-alexey@yandex.ru \\ P. Titov \\ Far-East National University E-mail: $\mathrm{t}-\mathrm{p}-1 \mathrm{e}$ inbox.ru
}

\section{A. Mihalyuk}

Far-East National University E-mail: mihalyuk.alexey@cis.pamho.net

\begin{abstract}
We offer the mathematical apparatus for mapping lattice and cellular systems into the generalized coordination Cayley's tree graphs. These Cayley's trees have random branchiness property and intralayer interbush local intersection. Classical Bethe-Cayley tree graphs don't have these properties. Bush type simplicial decomposition on Cayley's tree graphs is introduced, on which the enumerating polynomials or enumerating distributions are built. Within the entropy methodology a fractal characteristic in terms of Radon-Nikodym derivative is introduced, which characterizes quasi-crystalline pentagonal Penrose tiling.
\end{abstract}

The XXVII International Symposium on Lattice Field Theory - LAT2009

July 26-31 2009

Peking University, Beijing, China

\footnotetext{
*Speaker.
} 


\section{Introduction}

Since the discovery of quasicrystals by Shechtman and others [1], quasi-crystalline symmetries have become the subject of numerous investigations. If to talk about quasi-crystalline symmetries theory, then one of the major problems will be the order-disorder degree diagnostics.

According to the main tendencies of mathematical crystallography originated from the VoronoiDelaunay decompositions [2], paving, coating and partition procedures are considered in terms of absolutely solid tiles. The problem is to define which symmetry transformations are possible here. From our point of view it shouldn't be restricted only by the "tile's" alphabet in the problem of quasi-crystalline paving synthesis. It is necessary to take into account some direct coordinations, adjacency between the neighboring tiles. It is the coordination component of lattice structures that usually stands aside. In recent years some studies, which avoid the problem of tile's matching are appeared [3, 4, 5]. These papers may be related to the physical direction of quasicrystal growth problem, avoiding the "tile's formalism". Particularly in paper [5], authors use a coupled shortrange potential, which in the modeling process leads to the typical quasicrystal aperiodicity. The physical interpretation of this mechanism is given in paper [6]. It should be noticed that in paper [5] at the same time consideration via cellular structure of Penrose tiling boundaries is carried out. The icosahedral property, which is often used in the theory of amorphous solids we perceive as an extra excessive load in the model. Under the physical approach the integral criteria such as an average macrodensity of the filling out procedure or internal energy of growing cluster are prevail [5, 6]. Here is necessary to remember ideas of Penrose [7], that modeling of Monte-Carlo cluster forming will demand the introduction of relaxation procedures and integral rearrangement procedures for the whole cluster. The strategy of successive atoms conjunction with corresponding minimizing of growing cluster can not be the solution in the problem of quasicrystal structure synthesis. In our opinion, one should not eliminate the task of tile's matching and should not substitute for purely physical approach. It is necessary to give such representation of lattice, cellular structures, where both "tile's", and coordination components would be peer. Generalized tree Cayley graphs (TCG) are right the such objects.

\section{Informodynamical method for studying of cellular, lattice systems}

In our papers [8, 9, 10] a set of studies dedicated to searching the general representation method of quasi-crystalline lattices and cellular systems of mesodefects of quartz glasses and amorphous films was carried out. The formalism for mapping these objects into coordination tree Cayley graphs was also introduced there.

It is necessary to indicate three important points, which distinguish general TCG from BetheCayley tree graphs. The variable degree of branching of the bushes is accepted for such TCG. This fact in turn will be the indicator, that such coordination TCG may be endowed by probabilistic measures, distributions. Finally, the third and a quite important moment, is that our coordination TCG allow local interbush intralayer intersection. SuperTCG cannot be studied without applying decomposition methods. We chose the simplicial decomposition. Requirement of simpliciality is extremely important in the theory of complex systems [11]. This requirement in turn strongly correlates with the well-known Mandelbrot's fractality definition. 

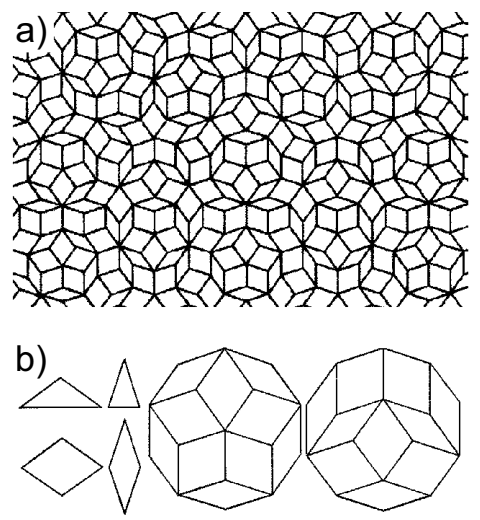

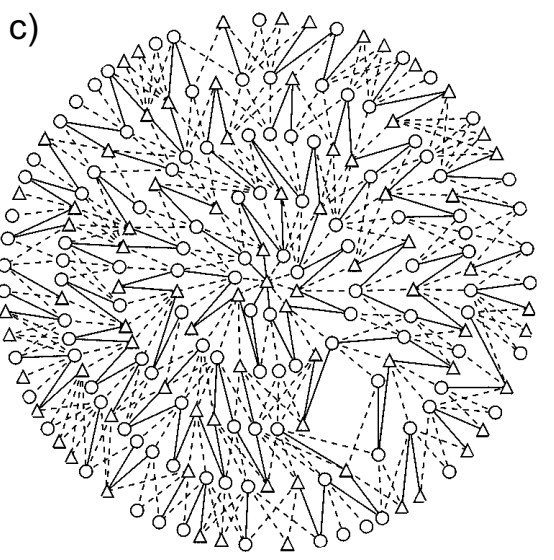

Figure 1: The Penrose tiling (a) with it's alphabet (b), and tree Cayley graph (c).

The coordination tree Cayley graphs (TCG) equally reflect both tiling part in their own vertices and direct coordinations (incidences) in their own edges. If to consider our superTCG, which represents lattice, cellular systems then we eo ipso achieve complete description of both tiling (element) component, and coordination component (ways of neighboring). That's why in our papers the conception of hierarchical alphabet consisting of the elementary typical modules is used. For example, in papers [9, 10] it was shown that for pentagonal Penrose symmetry the alphabet $[2 q \times 2 p]$ is sufficient. Figure 1 shows Penrose tiling (a), three-level hierarchy tile's alphabet (b), and it tree Cayley graph (c). In paper [7] this nonrecursive procedure was carried out at the second alphabet level. This procedure may be simplified by employing block coding conception from the information theory. Then synthesis of such lattices is realized by the third alphabet level (by the pair of decagons Fig. 1b), though minimal crossings by modules of second alphabet level have to be assumed here. Still, heuristics of quasi-crystalline mosaics synthesis at the third level of alphabet looks much simpler than nonrecursive procedure at the second alphabet level.

The mapping process of generalized lattices, and cellular systems into coordination tree Cayley graphs works as follows:

(i) Any module from the dual alphabet of second level is accepted as the TCG vertex. Then the rosette of initial coordinations, adjacencies, vicinities is being built.

(ii) From the reached vertices analogous straight vicinities directed only forward are built.

This cascade of coordinations in principle is infinite, thus we obtain growing TCG (Fig. 1c). All vertices and edges for Penrose mosaic may be described by alphabet $[2 q \times 2 p]$, where the qcoordinate indicates the vertex type (2-nd alphabet level), and the p-coordinate characterizes the straight contact with the other lower standing module. For the planar case two contact and vertex types are possible. Figure 18 shows the coordination TCG for the Penrose mosaic. For the planar case two contact and vertex types are possible.

Figure 10 shows the coordination TCG for the Penrose mosaic. By own topology it substantially differs from the widely known Bethe-Cayley tree graphs [12, 13]. In our method the variable (even random) branchiness degree of outgoing bushes is assumed. So even in this sense such TCGs are endowed by the appropriate probabilistic measures, distributions. Besides, our coordination TCGs have property of intralayer local interbush intersection, which is usually absent in the classi- 
cal Bethe and Cayley's tree graphs [12, 13]. TCGs are endowed by internal metrics (ultrametrics). Meanwhile the external metrics of the paving surface will be the Euclidean one. Then we have deal with non-Euclidean mapping of TCG onto plane with Euclidean topology. In goal to empathize abovementioned effect of intersection on the TCG, we introduced the term Markovian "jungles". One may equivalently present evolving TCG in the percolation terms. Any functionals on the TCG are the subject to percolation [8, 9, 10].

In general, as we already noticed, tree graph has the quasistochasticity property, and this factor is exclusively internal and not the externaly imported. In our case, in Fig. 1t one may readily see, that we have no deal with any stochasticity. This system seems not to be the stochastic, but the chaotic one. We want to especially emphasize the significant difference between stochasticity and chaos categories. Chaos may possess some ordering degree, which is observed in the corresponding fractal dimension values. Shown in Fig. 1e TCG may be described by conventional forming logic:

$$
G=\left\{N=\sum_{i} \sum_{j_{i}} n_{j_{i}} ; \bigcup_{i}^{n_{i}} \bigcup_{j_{i}}^{n_{j_{i}}} x\left(l_{j_{i+1}} / l_{j_{i}}\right) ; K\left(l_{j_{i}}\right)\right\}
$$

where $i$ - is the TCG level, $j_{i}$ - is the level index of $i$-level, $K\left(l_{j_{i}}\right)$ - is the branchiness index of $j_{i}$-vertex at the $i$-level, $x\left(l_{j_{i+1}} / l_{j_{i}}\right)$ - is the outgoing edge from $j_{i}$ to $j_{i+1}$ (conventional logic), $n_{j_{i}}-$ are the vertices at the $i$-level, $N$-is the total quantity of TCG vertices, $\bigcup_{i} \bigcup_{j_{i}}$ - are the consolidation operators by $j_{i}$-vertices and $i$-levels.

Let us empathize that in Eq. (2.1) edges are presented in the conventional logic, where $j_{i}-$ is a starting index, and edge is directed to the address $j_{i+1}$. Like for any graph, in Eq. (2.1) vertices are described, and the most important - the branchiness degree of outgoing bush is defined. This term (bush) prompts that in the process of tree graph analysis appropriate decomposition methods must be applied, among which we especially empathize the simplicial decomposition [11, 14]. One may conveniently realize it in the bush approach. Any bush may be presented as the sum of outgoing branches:

$$
g_{x}\left(K\left(l_{j_{i}}\right)\right) \equiv \sum_{j_{i+1}}^{K\left(l_{j_{i}}\right)} x\left(l_{j_{i+1}} / l_{j_{i}}\right)
$$

In Eq. (2.2) the partial bush with branchiness $\left(l_{j_{i}}\right)$ is considered, which starts from the corresponding vertex. The summation procedure is accomplished for partial bush by $j_{i+1}$ index. $x\left(l_{j_{i+1}} / l_{j_{i}}\right)$ - are branches in the conventional logic. Consequently the TCG (Eq. (2.1) ) has to be decomposed into simplicial bush decomposition:

$$
G=\left\{N ; \sum_{i}^{n_{i}} \sum_{j_{i}}^{n_{j_{i}}} g_{x}\left(K\left(l_{j_{i}}\right)\right)\right\}
$$

Equation (2.3) in comparison with Eq. (2.1) is a considerable advancement, because here hierarchical bush decomposition is used. The Markovian expansion is allowed by $i$-index, i.e. we deal with the radial M-chain. It should be noted that Markovian feature in the tangential direction is not satisfied since local interbush intralayer intersection property presence. On the lattice, cellular structures the flow of coordinations is the subject to percolation, this flow is topologically reflecting in the growing TCG. 
The conception of simplicial decomposition in fact means the decomposition of superTCG into bushes (Eq. (2.3)). Taking into account the character of TCG internal metric, we will introduce the ultrasimplex concept, which interprets the partial bush. Therefore simplicial decomposition of TCG may be treated as the method of hierarchical decomposition of supersimplexes into additive composition of ultrasimplexes. This principle is quite constructive, because automatically assumes the topological similarity of supersimlex and its constituent modules - partial ultrasimplexes. Such approach is known in the system analysis of complex structures [11]. From the other side, one may remember the fractal methodology [15], where the fact of decomposition of big complex system into similar in certain criteria modules is the necessary requirement for the fractality.

Further inquiry of superTCG is based on the applying of enumerative theory [14] to the treegraph structures. In the classical approach enumerating polynomials (EP) are calculated. It may be specified for each tree hierarchy level:

$$
\Pi_{i}\left(x^{r}\right)=\sum_{k}^{r} T_{i}\left(x^{k}\right) \cdot x^{k}
$$

Any EP is specified by two freedom degrees: $K$ - is the bush branchiness degree, $T_{i}\left(x^{k}\right)-$ are coefficients of EP in the absolute form. Coefficients point to the number of bushes with fixed branchiness at the corresponding TCG level. It should be noted that EP in that form assumes the algebraic direction of graph's studying.

Further, when appropriate normalization procedures are accomplished one may proceed from EP to probabilistic EP (PEP), and consider these PEP as probabilistic distributions (already no polynomials):

$$
\Pi_{i}\left(x^{r}\right)=\sum_{k}^{r} t_{i}\left(x^{k}\right) \cdot x^{k} ; t_{i}\left(x^{k}\right)=\frac{T_{i}\left(x^{k}\right)}{\sum_{k}^{r} T_{i}\left(x^{k}\right)}
$$

In that case one may consider the percolation of such statistics on the TCG. The opportunity of studying statistical forms of percolation is opening up here. The theory of dynamical chaos supposes that systems of such kind may be analyzed by statistical methods, in spite of their "dynamics". The next stage in the informodynamical method $[8,9,10]$ is the proceeding to the function level. Here one has to compute functionals over EP, PEP and to consider their percolation on the TCG. We hope that percolation dependencies of entropy functional [16] will give the opportunity to identify the order-disorder character of generalized lattices and cellular systems.

$$
H_{v}\left[\Pi_{i}\left(x^{r}\right)\right]=\sum_{k}^{r} t_{i}\left(x^{k}\right) \cdot\left[1-t_{i}\left(x^{k}\right)\right]=\sum_{k}^{r} t_{i}\left(x^{k}\right) \cdot \overline{t_{i}\left(x^{k}\right)}
$$

Above formulated principle of superTCG simplicial decomposition immediately prompts a quite strong correlation with the fractality conception [15]. As known, at the basis of fractality conception the scaling property is the necessary condition. The simplicial decomposition automatically possesses this property, because bushes are the subtrees in the topological plane. Tree-graph approach gives a new opportunity to formulate the method of fractal dimension estimation for quasi-crystalline mosaics, tilings, cellular systems of mesodefects [8, 17]. In the fractal theory the dimension principle of Hausdorff-Besicovitch methodology is used, and in recent years a great number of different algorithms for estimation of fractal characteristics were offered. In this paper, 
fractality estimation methods for tree-graph structures occupy the central position. We will be stating them in entropy terms. These freedom degrees are EP, PEP coefficients and bush branchiness degrees. Just for them we offer to define the entropy measures. Eo ipso we get the opportunity to introduce functions in the Radon sense [18] - the dependence of entropy of EP, PEP coefficients from the entropy of ultrasimplex average bush branchiness. Continuing this way one may employ the Radon-Nikodym derivative concept, and in the simplest case to demand its consistency, which in turn will be the corresponding fractal characteristic [18].

\section{Tangential fractal dimension for Cayley's tree graph of the Penrose tiling}

Now we will give the fractal dimension estimate for Penrose tiling in the tree-graph representation employing the supremum characteristics of percolation of enumerating structures on the TCG. Particularly the enumerating polynomial (EP) may be taken as the basis for estimation method. EP is characterized by $r_{i}$ - polynomial rank, $T_{i}\left(x^{k}\right)$ - EP coefficient, and $k_{i}$ - level of bush branchiness. Here we just give the final expression for fractal dimension in approaching of outgoing bushes:

$$
d_{t g}\left(T_{\Sigma^{10}}\left(x^{k}\right)\right)=\frac{1}{r_{C P}\left(T_{\Sigma^{10}}\left(x^{k}\right)\right) \cdot \ln i_{\Sigma^{10}}} \cdot \sum_{k=0}^{r\left(T_{\Sigma^{10}}\left(x^{k}\right)\right)} \frac{\ln \left(T_{\Sigma^{10}}\left(x^{k}\right)\right)}{\ln (k+2)}
$$

where $i \geq 2 ; 0 \leq k \leq r, r_{i}$ - is a polynomial rank; $T_{i}\left(x^{k}\right)$ - is a EP coefficient; $x^{k}-$ is a bush with $k$-branchiness at $i$-level; $\ln i_{\Sigma^{10}}-$ is a ultraheight for 10-th TCG level. The total sum of independent outgoing bushes at any TCG level we consider as a tangential phrase. Symbols of such phrases are bushes with corresponding branchiness degrees. The linguistic approach is useful for considering the multifractal objects. Than under the summation symbol of Eq. (3.1) stands the ratio of entropy of bush reiteration to the entropy of bushes itself. The summation process is going by $k$ up to EP rank value. In such case, the tangential phrase is the sum of normalized entropy functionals. Alternatively, the number of bush quantums expresses the quantity of diversity in the tangential phrase. Normalizations by EP rank and ultraheight of TCG are performed further. Numerical estimate for our TCG of Penrose tiling for $T_{\Sigma^{10}}\left(x^{k}\right)$ according to abovementioned assumptions yields:

$$
d_{t g}\left(T_{\Sigma^{10}}\left(x^{k}\right)\right)=(16.118 \ldots)^{-1} \cdot 22.19 \ldots \approx 1.38
$$

Obtained estimate (Eq. (3.2)) for tangential fractal dimension of TCG of Penrose tiling is interpreted as a capacity of percolation. This estimate is the supremum one, since is obtained in the approaching of outgoing independent bushes for each level of TCG hierarchy. In our papers [8, 9, 10] and in paper [19] the fractal dimension of Fibonacci system, which yields 0.6909 was calculated. If in paper [19] the fractal dimension was calculated by the Hausdorff-Besicovitch methodology, then in our studies it was calculated by the informodynamical method.

The Fibonacci symmetry is essentially inherent to the pentagonal Penrose tiling. The percolation task of tangential fractality on the TCG of the Penrose tiling has been also analyzed on the level of tangential fractality. Figure 2 shows a quite confident wavelike oscillatory percolation dependence of the tangential fractality. This supremum capacitive feature is quite important, therefore, it is necessary to calculate the estimate of fractal dimension by one additional technique. According to the enumerative tree graph theory [14], we will assume normalized EP or probabilistic EP and 


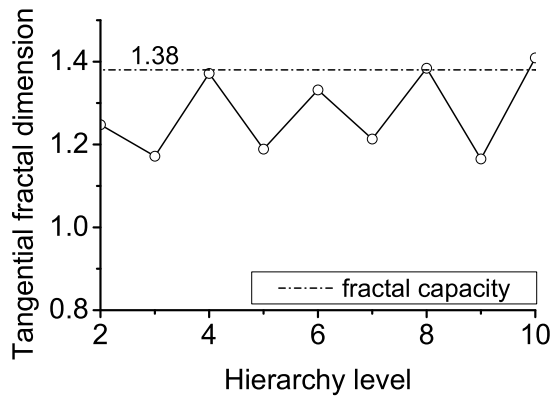

Figure 2: The percolation of tangential fractal dimension on the tree Cayley graph of the Penrose tiling.

corresponding entropy functionals in averaged or asymptotic versions as the methodological basis. It is possible to obtain weaker supremum estimate under such conditions. The final equation is:

$$
\begin{gathered}
d_{t g}\left(t_{\Sigma^{10}}\left(x^{k}\right) ; K_{\infty} \vee \bar{K}_{10}\right)=\frac{\sup \left[H\left(t_{\Sigma^{10}}\left(x^{k}\right)\right)\right]}{\ln \left[\left(K_{\infty} \vee \bar{K}_{10}\right)+1\right]}, \\
d_{t g}\left(t_{\Sigma^{10}}\left(x^{k}\right) ; \bar{K}_{10}\right)=1.4078 \ldots,
\end{gathered}
$$

where $t_{\Sigma^{10}}\left(x^{k}\right)$ - is a probabilistic EP (or bushes distribution by branchiness factor) for cumulant up to the 10-th level; $K_{\infty} \vee \bar{K}_{10}$ - are the asymptotic or normalized average branchiness on the TCG.

In the numerator of Eq. (3.3) the upper limit of entropy is located, which in turn is divided to the entropy of average or asymptotic branchiness. Notice that this equation is quite different from the Eq. (3.1), but construction logic is the same for both equations. In this case the informationtheoretical interpretation may be given, which is free from the service specific normalizations in Eq. (3.1). The fractal dimension is obtained as a ratio of entropy to the entropy of bush branchiness degrees either in asymptotic or in average. These upper fractal estimates may be interpreted as linearized form of derivative in the Radon-Nikodym sense [18].

\section{Conclusion}

We have offered an adequate and one-one mapping of lattice structures into coordination tree Cayley graphs. Such representation allows describing the coordination component, or the way of cell interaction of the cellular system in an explicit way. Complex systems have to be represented in the tree-graph hierarchy, which is realized by the generalized Bethe-Cayley graphs. We suggest to study graphs by method of simplicial bush decomposition. Generally, the bush is similar to the supertree, which is the necessary fractality condition. After performing a simplicial bush decomposition of TCG, at the each hierarchy level the enumerating polynomials and different functionals over them are build, among which we mark out the informational entropy. The symmetry of TCG and ordering of cellular structure are studied by analyzing percolation of functional dependencies by the hierarchy levels. If to consider quasicrystals, then the graph itself is determined. In analysis of internal percolation task on TCG the entropy invariants in average with oscillation-wave behavior were identified. According to tree-graph formalism, we have deal with principle of topological 
tree scaling. This fact allows introducing fractal characteristics on TCG, which represent the percolation of functionals through system of corresponding fronts and duals to them rays. An estimation of fractal dimension in our method is performed via Radon functions, which are the coefficients of EP entropy as a function of TCG ultraheight. Thereby any fractal dimension is a derivative in a sense of Radon-Nikodym.

\section{References}

[1] D. Shechtman, I. Blech, D. Gratias, and J. W. Cahn, Phys. Rev. Lett. 53, 1951 (1984).

[2] A. V. Anikeenko, N. N. Medvedev, and T. Aste, Phys. Rev. E 77, 031101 (2008).

[3] P. J. Steinhardt and H.-C. Jeong, Nature (London) 382, 431 (1996).

[4] P. J. Steinhardt, H.-C. Jeong, K. Saitoh, M. Tanaka, E. Abe, and A. P. Tsai, Nature (London) 396, 55 (1998).

[5] A. S. Keys and S. C. Glotzer, Phys. Rev. Lett. 99, 235503 (2007).

[6] P. J. Steinhardt, Nature (London) 452, 43 (2008).

[7] R. Penrose, Emperor's New Mind (Oxford University Press, New York, 2002).

[8] V. V. Yudin, T. A. Pisarenko, E. A. Lyubchenko, and E. G. Savchuk, Crystallogr. Rep. 44, 373 (1999).

[9] V. V. Yudin and Yu. A. Karygina, Crystallogr. Rep. 46, 922 (2001).

[10] V. V. Yudin, T. A. Pisarenko, E. A. Lyubchenko, O. A. Chudnova, and Yu. A. Karygina, Crystallogr. Rep. 47, 189 (2002).

[11] J. L. Casti, Connectivity, Complexity, and Catastrophe in Large-scale Systems, (John Wiley \& Sons, New York, 1979).

[12] G. Paul and H. E. Stanley, Phys. Rev. E 67, 026103 (2003).

[13] T. Brookings, J. M. Carlson, and J. Doyle, Phys. Rev. E 72, 056120 (2005).

[14] F. Harari and E. M. Palmer, Graphical Enumeration (Academic Press, New York, 1973).

[15] H.-O. Peitgen, H. Jurgens, D. Saupe, Chaos and Fractals (Springer-Verlag, New York, 2004).

[16] N. F. G. Martin and J. W. England. Mathematical Theory of Entropy (Addison-Wesley Publishing Company, London, 1981).

[17] V. V. Yudin, Stochastic Magnetic Structure of Films with Microporous System (Nauka, Moscow, 1987) (in Russian).

[18] R. E. Edwards, Functional Analysis. Theory and Applications (Holt, Rinehart and Winston, New York, 1965).

[19] A. I. Olemskoy and A. Ya. Flat, Phys. Usp. 36, 1087 (1993). 